\title{
Myth: Parenteral ketorolac provides more effective analgesia than oral ibuprofen
}

\author{
Sanjay Arora, MD; ${ }^{*}$ Jonathan G. Wagner, BA; ${ }^{\dagger}$ Mel Herbert, MD, MB BS, BMedSci ${ }^{\ddagger}$
}

\section{Introduction}

Acute pain is an extremely common presenting symptom to the emergency department (ED), making it imperative that emergency physicians provide adequate, safe and cost-effective analgesia. Nonsteroidal anti-inflammatory drugs (NSAIDs) are often first-line treatments for moderate to severe pain. Physicians can choose between intramuscular (IM) or intravenous (IV) ketorolac and an oral NSAID. The mechanism of action (reversible inhibition of prostaglandin synthesis at the level of cyclooxygenase) is identical irrespective of the route the medication is given. ${ }^{1}$ Despite the similar pharmacodynamics, many physicians believe that parenteral ketorolac is more efficacious, despite a greater cost and a more invasive route of administration. To investigate this myth (i.e., that parenteral ketorolac provides greater analgesic effect than an oral NSAID), we conducted a review of the literature, with specific focus on ibuprofen as the prototypical — and least expensive — oral NSAID.

\section{Methods}

The terms "ketorolac" and "ibuprofen" were searched in MEDLINE and PubMed, revealing 17 and 67 articles, respectively. Articles were limited to English language and those involving human subjects. All abstracts were reviewed. Articles directly comparing oral ibuprofen with IM or IV ketorolac were included. To ensure no important papers were missed, an ancestral search of identified articles was also performed.

\section{Results}

In 1994, Wright and associates evaluated the effectiveness of a single dose of $800 \mathrm{mg}$ of oral ibuprofen $(n=95)$ versus $60 \mathrm{mg}$ of IM ketorolac $(n=70)$. This was a retrospective analysis of data collected during a prior prospective survey of pain management efficacy, in patients presenting to the ED with acute pain due to a large variety of causes. ${ }^{2}$ Using the 100-mm visual analog pain scale (VAS) they found a mean score reduction of 34 in the ibuprofen group and 35 in the ketorolac group. They concluded that the 2 have almost identical efficacy in those presenting with acute pain of varied sources, and that unless oral administration is contraindicated, ibuprofen is superior given its ease of administration, its significantly lower cost, and the lack of pain associated with administration. ${ }^{2}$

In 1995, Turturro and colleagues compared $800 \mathrm{mg}$ of oral ibuprofen with $60 \mathrm{mg}$ of IM ketorolac in a prospective, double-blind, randomized trial of 82 patients presenting to the ED with acute traumatic musculoskeletal pain. ${ }^{3}$ They used the 100-mm VAS to quantify pain at baseline, 15, 30, 45, 60, 75, 90 and 120 minutes post dosing. They found no significant differences in mean pain scores at baseline or at any time point throughout the 2-hour period. They noted that the ibuprofen group exhibited lower mean pain scores in the later intervals of the study; however, it was not statistically different. They concluded that oral ibuprofen and IM ketorolac provide similar analgesia with similar onset of action in minor-to-moderate acute musculoskeletal pain and reasoned that IM ketorolac should be reserved for those

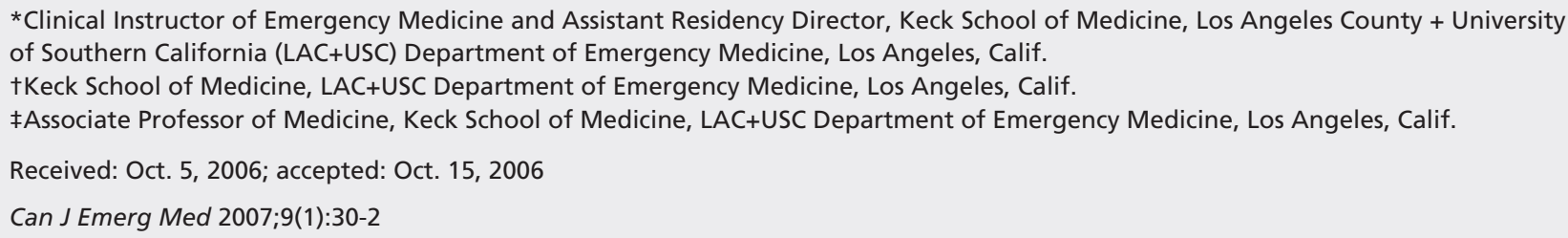


patients with contraindications to oral intake due to its painful administration and its higher cost (170 times that of ibuprofen at their institution at the time of the study). ${ }^{3}$

In yet another prospective, randomized, double-blind study in the ED, Neighbour and Puntillo investigated the analgesic efficacy of $60 \mathrm{mg}$ of IM ketorolac versus $800 \mathrm{mg}$ of oral ibuprofen in patients with self-assessed pain scores of $5,6,7$ or 8 on a numerical rating scale of $0-10$ ( 0 corresponding to "no pain" and 10 corresponding to "worst possible pain") for a variety of pain etiologies. ${ }^{4}$ Patients' pain levels were assessed at $0,15,30,45,60,90$ and 120 minutes after administration of analgesic. They found no statistical differences in pain levels between study groups at any time point in the study, further emphasizing conclusions reached in the 2 prior ED studies.

In a 1998 prospective, randomized, double-blind study conducted in surgical patients, Mixter and coworkers investigated the use of $60 \mathrm{mg}$ of IV ketorolac at the time of trocar insertion versus $800 \mathrm{mg}$ of oral ibuprofen 1 hour before surgery in laparoscopic hernia repairs. ${ }^{5}$ All patients were discharged within 5 hours of completion of surgery, and patients were instructed to take ibuprofen $400 \mathrm{mg}$ orally every 4 hours for 24 hours regardless of pain level. They measured pain using the 100-mm VAS at time of discharge and found no significant difference between the ketorolac group (VAS = 35 ) and the ibuprofen group (VAS = 30). They also measured pain 18 hours after discharge, and found an identical score in the 2 groups (VAS $=20$ ). Like the previous ED studies comparing the 2 drugs, the authors concluded that oral ibuprofen offers equal pain control at lower cost and reduced potential for adverse drug events for post-surgical patients. ${ }^{5}$

Interestingly, in one of the first studies comparing ketorolac with ibuprofen in post-op patients, Morrison and Repka did find a significant difference in pain control. ${ }^{6}$ In this prospective, randomized, double-blind study, they compared a single dose of $60 \mathrm{mg}$ of IV ketorolac intraoperatively $(n=$ 20) with a single dose of $600 \mathrm{mg}$ of ibuprofen $30-45 \mathrm{~min}$ utes after completion of strabismus surgery $(n=20)$. Pain was assessed with the 100-mm VAS. At 2 hours post-op, the VAS for ketorolac patients was 15 and for the ibuprofen patients it was 50 . At 5 hours post-op, the VAS for ketorolac patients was 20 and for the ibuprofen patients it was 44 . They concluded that IV ketorolac was more effective than oral ibuprofen in controlling postoperative pain in patients undergoing strabismus surgery. However, the study design needs further scrutiny before taking this data at face value: there were multiple methodological flaws, and the results, as the authors themselves point out, should be interpreted with caution. ${ }^{6}$ In their study, the oral ibuprofen was not distributed in its commercially available formulation; instead, it was crushed and administered in unmarked capsules, which may have altered the analgesic's pharmacodynamics. Even more importantly, in the protocol IV ketorolac was given at least 30-45 minutes before the oral ibuprofen was administered. Multiple studies have shown that as patients receive repetitive painful stimuli over time without analgesia, the overall total perception of pain significantly increases. ${ }^{7.8}$ Those patients in this study receiving oral ibuprofen late in the course of their care had time to suffer from this so-called "wind-up" phenomenon, which is a complex neurotransmitter-based phenomenon that results in "pain begetting pain." It is impossible to draw conclusions from a study comparing the efficacy of 2 drugs when the oral format was given well after the IV preparation. A balanced study would instead have allowed the oral format to be given prior to the IV one.

\section{Discussion}

The studies discussed in this paper dealt with patients who had a wide variety of pain syndromes, but there is some anecdotal evidence and a limited number of published studies touting the efficacy of parenteral ketorolac when used specifically for the treatment of renal colic. It has been shown in multiple studies that ketorolac is as good as, if not better than, low dose meperidine in providing effective pain relief in renal colic. ${ }^{10-14}$ Similar results were found in studies comparing the efficacy of ketorolac with diclofenac in patients with renal colic. ${ }^{15,16}$ Safdar and colleagues compared ketorolac to morphine for the treatment of acute renal colic using a prospective randomized, controlled, double-blinded design. ${ }^{17}$ Patients received either $5 \mathrm{mg}$ of IV morphine or 15 $\mathrm{mg}$ of IV ketorolac at time zero with a repeat dose of the same at 20 minutes. A third group received both agents at both time intervals. They found no significant difference in efficacy between the ketorolac and morphine groups in relief of pain, but did find a significant reduction in pain in the combination group. They therefore concluded that a combination of morphine and ketorolac provides greater analgesia than using either of the agents alone. ${ }^{18}$ Despite the plethora of trials comparing ketorolac with narcotics and proving the utility of NSAIDs for the treatment of renal colic, there are no identifiable studies comparing parenteral ketorolac with oral ibuprofen in this setting. It is quite possible that, as in the treatment of other acute pain syndromes, ibuprofen may provide equal analgesia. Given that vomiting is frequently a part of the presentation of renal colic, a parenteral NSAID may be preferred.

Many physicians continue to administer IM or IV ketorolac regardless of the aforementioned studies, perhaps due to the belief that patients respond better to parenteral analgesia 
because the patients consider them "stronger" medicines. Schwartz and associates investigated this perceived placebo effect in a fascinating prospective, randomized, doubleblind study in which patients were unknowingly given 800 $\mathrm{mg}$ oral ibuprofen in a flavoured drink and then given either a placebo IM injection or a placebo pill. In this interesting study design, neither group received IM medication. They found no significant difference in pain reduction via the VAS and concluded that the use of IM administration of NSAIDs for pure placebo effect appears unwarranted..$^{18}$

In addition to a lack of improved efficacy and no benefit from a placebo injection effect, there are potentially serious downsides to this tactic. First, there is the risk of a needlestick injury to health care personnel from unnecessarily using a parenteral medication when an oral form will work just as well. Second, even though all NSAIDs have the potential to exacerbate renal dysfunction, parenteral ketorolac seems particularly potent in this regard. In fact, in several European countries the 60 -mg form of ketorolac was taken off the market due to its association with acute renal failure. It is also interesting to note that the manufacturer suggests that the oral dose of ketorolac be $10 \mathrm{mg}$ but the parenteral form be 30-60 mg. Which other drug has a higher dose when given parenterally than orally? The exact implications of this dosing conundrum remain unclear, although an unacceptably high risk of gastrointestinal adverse events is felt to be the root cause of the lower oral dose.

\section{Conclusion}

The higher cost of ketorolac, the pain and difficulty associated with its administration, the risk of extravasation, and the exposure of practitioners to possible needle-stick injuries, all argue that there is no use for IM or IV ketorolac over oral ibuprofen in the ED for routine analgesia, unless oral administration of ibuprofen is unfeasible or contraindicated. Only in specific acute pain syndromes associated with nausea and vomiting, like renal colic, may its use be warranted. The belief that IM/IV medications are perceived as being stronger than oral medications and therefore result in a more powerful placebo effect has also been shown to be false. With the exception of 1 study in post-op patients with a significantly flawed study design, the evidence overwhelmingly shows that inexpensive and relatively safe oral ibuprofen has equal efficacy to the more expensive and potentially dangerous IM or IV ketorolac. ${ }^{2-6}$

\section{Competing interests: None declared.}

Disclosures: Dr. Herbert owns and edits EM:RAP and CMEdown load.com, a Web site that includes online EM education products.
Key words: ketorolac; ibuprofen; analgesia; acute pain

\section{References}

1. Buckley MM, Brogden RN. Ketorolac: a review of its pharmacodynamic and pharmacokenetic properties, and therapeutic potential. Drugs 1990;39:86-109.

2. Wright JM, Price SD, Watson WA. NSAID use and efficacy in the emergency department: single doses of oral ibuprofen versus intramuscular ketorolac. Ann Pharmacother 1994;28:309-12.

3. Turturro MA, Paris PM, Seaberg DC. Intramuscular ketorolac versus oral ibuprofen in acute musculoskeletal pain. Ann Emerg Med 1995;26:117-20.

4. Neighbor ML, Puntillo KA. Intramuscular ketorolac vs. oral ibuprofen in emergency department patients with acute pain. Acad Emerg Med 1998;5:118-22.

5. Mixter CG 3rd, Meeker LD, Gavin TJ. Preemptive pain control in patients having laparoscopic hernia repair: a comparison of ketorolac and ibuprofen. Arch Surg 1998;133:432-7.

6. Morrison NA, Repka MX. Ketorolac versus acetaminophen or ibuprofen in controlling postoperative pain in patients with strabismus. Ophthalmology 1994;101:915-8.

7. Price DD, Hu JW, Dubner R, et al. Peripheral suppression of first pain and central summation of second pain evoked by noxious heat pulses. Pain 1977;3:57-68.

8. Arendt-Neilson L, Brennum J, Sindrup S, et al. Electrophysical and psychophysical quantification of temporal summation in the human nociceptive system. Eur J Appl Physiol 1994;68:266-73.

9. Guirimand F, Dupont X, Brasseur L, et al. The effects of ketamine on the temporal summation (wind-up) of the R(III) nociceptive flexion reflex and pain in humans. Anesth Analg 2000;90:408-14.

10. Larsen LS, Miller A, Allegra JR. The use of intravenous ketorolac for the treatment of renal colic in the emergency department. Am J Emerg Med 1993;11:197-9.

11. Sandhu DPS, Iacovou JW, Fletcher MS, et al. A comparison of intramuscular ketorolac and pethidine in the alleviation of renal colic. Br J Urol 1994;74:690-3.

12. Cordell WH, Wright SW, Wolfson AB, et al. Comparison of intravenous ketorolac, meperidine, and both (balanced analgesia) for renal colic. Ann Emerg Med 1996;28:151-8.

13. Oosterlinck W, Philp NH, Charig C, et al. A double-blind single dose comparison of intramuscular ketorolac tromethamine and pethidine in the treatment of renal colic. J Clin Pharmacol 1990; 30:336-41.

14. Larkin GL, Peacock WF IV, Pearl SM, et al. Efficacy of ketorolac tromethamine versus meperidine in the ED treatment of acute renal colic. Am J Emerg Med 1999;17:6-10.

15. Cohen E, Hafner R, Rotenberg Z, et al. Comparison of ketorolac and diclofenac in the treatment of renal colic. Eur J Clin Pharmacol 1998;54:455-8.

16. Stein A, Ben Dov D, Finkel B, et al. Single-dose intramuscular ketorolac versus diclofenac for pain management in renal colic. Am J Emerg Med 1996;14:385-7.

17. Safdar B, Degutis LC, Landry K, et al. Intravenous morphine plus ketorolac is superior to either drug alone for treatment of acute renal colic. Ann Emerg Med 2006;48:173-81.

18. Schwartz NA, Turturro MA, Istvan DJ, et al. Patients' perceptions of route of nonsteriodal anti-inflammatory drug administration and its effect on analgesia. Acad Emerg Med 2000;7:857-61.

Correspondence to: Dr. Sanjay Arora, Department of Emergency Medicine, Los Angeles County-USC Healthcare Network, 1200 N State St., Los Angeles CA 90033 